PROCEEDINGS OF THE

AMERICAN MATHEMATICAL SOCIETY

Volume 106, Number 4, August 1989

\title{
MAXIMUM RANK OF POWERS OF A MATRIX OF A GIVEN PATTERN
}

\author{
SVATOPLUK POLJAK
}

(Communicated by Andrew M. Odlyzko)

\begin{abstract}
The pattern of a matrix is the structure of its zero and nonzero entries. For any prescribed pattern we determine the maximum possible rank of each power of a matrix with a given pattern. The approach is based on a combinatorial result that may be of some interest independently.
\end{abstract}

\section{INTRODUCTION}

The pattern of a matrix is the structure of its zero and nonzero entries. In the well-known paper [2] J. Edmonds has shown that the maximum possible (linear algebra) rank of a matrix of a given pattern equals its term rank which is the maximum number of nonzero entries no two of which lie in a common row or column. The term rank equals the rank provided the nonzero entries are algebraically independent transcendentals. Let us recall one important consequence. Matrices arising in several applications are often sparse and without expected numerical dependencies among the entries, and hence one may assume that their rank equals the term rank. The term rank of a matrix can be computed by the matching algorithm for bipartite graphs [5]. Thus, there is an efficient algorithm for testing the rank of a matrix that does not depend on the numerical entries but only on the pattern.

However, in some applications, such as in automatic control, one needs to know also the ranks of the powers of a matrix. The term rank does not give a good upper bound for the powers. It may even happen that the term rank of powers is increasing while the maximum possible rank is decreasing. In this paper we determine the maximum rank of powers of a matrix of a given pattern. The result is based on a combinatorial theorem that can be interpreted as follows. Let $G$ be a digraph and $p$ a positive integer. Let $k$ be the maximum number of people who can be located in distinct vertices so that they can simultaneously walk for $p$ time units-traversing one edge per time unit-and

Received by the editors December 29, 1987 and, in revised forms, July 5, 1988 and October 10,1988. Presented at the 13th International Symposium on Mathematical Programming, Tokyo, Japan, August 29-September 2, 1988.

1980 Mathematics Subject Classification (1985 Revision). Primary 05C50.

(C)1989 American Mathematical Society $0002-9939 / 89 \$ 1.00+\$ .25$ per page 
two or more people never meet in a vertex. The result is that the schedule can always be organized in the following special way. One can find a collection of pairwise vertex disjoint cycles (of arbitrary length) and paths of length at least $p$, so that $k$ people are initially located in the vertices of the cycles and paths but not in the last $p$ vertices of each path. The people then walk for $p$ time units either around the cycle or along the path.

The number $k$ in the above schedule is the maximum rank of the $p$ th power of a matrix whose pattern is represented by $G$.

\section{RESULTS}

We use some basic notions of graph theory [1]. Let $G=(V, E)$ be a fixed digraph with possible loops and without multiple edges where $V=\{1,2, \ldots, n\}$ and $E$ is a set of directed edges. A walk $w=\left(v_{0}, e_{1}, v_{1}, \ldots, v_{p-1}, e_{p-1}, v_{p}\right)$ is a sequence of (not necessarily distinct) vertices and edges of $G$ so that $e_{i}=v_{i-1} v_{i}$ is a directed edge for each $i$. The number $p$ in the definition is called the length of the walk. A p-walk is a walk of length $p$. A path (a cycle) is a walk whose vertices are mutually distinct (but $v_{0}=v_{p}$ for a cycle). The number of vertices of a path $P$ or a cycle $C$ will be denoted by $|P|$ and $|C|$, resp. We say that two $p$-walks $w$ and $w^{\prime}$ are (vertex) independent if $v_{i} \neq v_{i}^{\prime}$ for $i=0,1, \ldots, p$ where $v_{i}$ and $v_{i}^{\prime}$ denote the $i$ th vertices of the walks.

For each positive integer $p$ we associate with $G$ another acyclic digraph $N(G, p)$, which will be called a network. The vertex set of $N(G, p)$ consists of $p+1$ copies $i(0), i(1), \ldots, i(p)$ of each vertex $i$ of $G$, and the edges are $i(t-1) j(t), t=1, \ldots, p$, for each edge $i j$ of $G$. Obviously, there is a 1-1 correspondence between the $p$-walks in $G$ and the paths of length $p$ in $N(G, p)$, and independent $p$-walks correspond to vertex disjoint paths.

The proof of the following Theorem 1 is based on linear programming. We formulate a linear program that solves packing of cycles and paths with respect to an objective function. Then we derive from the dual solution a vertex cut in $N(G, p)$ which bounds the number of vertex disjoint $p$-paths.

As a reference to linear programming and the theory of totally unimodular matrices we recommend the book [7].

Theorem 1. For every digraph $G$ and a positive integer $p$ there are mutually vertex disjoint cycles and paths $C_{1}, \ldots, C_{r}$ and $P_{1}, \ldots, P_{s}$ such that the maximum number of independent p-walks in $G$ equals

$$
\sum_{i=1}^{r}\left|C_{i}\right|+\sum_{i=1}^{s}\left(\left|P_{i}\right|-p\right) .
$$

Proof. We first show that the packing problem,

"Find a collection $\mathscr{F}$ of mutually vertex disjoint paths and cycles for which the value of (1) is maximum" 
can be formulated as a linear program. Let $K_{n}$ be the complete digraph on vertex set $\{1, \ldots, n\}$. Let us associate a weight $w_{i j}$ with each edge where

$$
\begin{array}{cl}
w_{i j}:= & \text { for } i j \in E, \\
1-p & \text { for } i j \notin E, i \neq j, \\
0 & \text { for } i \notin \notin E, i=j .
\end{array}
$$

We claim that the packing problem (2) is equivalent with the following problem.

$$
\text { "Find a collection } \mathscr{C} \text { of vertex disjoint cycles in } K_{n} \text { of the }
$$
maximum weight $w(\mathscr{C})$ where

$$
w(\mathscr{C}):=\sum_{C \in \mathscr{C}} w(C):=\sum_{e \in C \in \mathscr{C}} w_{e} . "
$$

Let us first observe that an optimum solution of (2) yields a feasible solution of (4) with the same cost. (The weight of a cycle is $w(C)=|C|$, and $w(P \cup e)=$ $|P|-p$, where $e \in K_{n} \backslash E$ is the edge that completes a path $P$ to a cycle.) Conversely, let $\mathscr{C}$ be an optimum solution of (4). Deleting all edges of $K_{n} \backslash E$ we obtain a collection of vertex disjoint paths and cycles with the same cost. (If a cycle $C$ contains $k$ edges of $K_{n} \backslash E$ then $C$ splits into $k$ paths.) This concludes the proof of the claim.

We may require, without loss of generality, that an optimum solution of (4) covers all vertices of $K_{n}$. If not, we can add the loop $i i$ with $w_{i i}=0$ for each uncovered vertex $i$. In other words, (4) is equivalent to

$$
\begin{aligned}
& \text { "Find a permutation } \pi \text { on }\{1, \ldots, n\} \text { for which } \sum_{i=1}^{n} w_{i, \pi(i)} \\
& \text { is maximum." }
\end{aligned}
$$

Problem (5) is known as the assignment problem, and it is solvable in polynomial time by a combinatorial algorithm. It is also well known that the assignment problem can be formulated as the following linear program.

$$
\max \sum w_{i j} x_{i j}
$$

subject to

$$
\begin{array}{ll}
\sum_{j=1}^{n} x_{i j}=1, & i=1, \ldots, n, \\
\sum_{i=1}^{n} x_{i j}=1, & j=1, \ldots, n, \\
x_{i j} \geq 0, & i, j=1, \ldots, n .
\end{array}
$$

Linear program (6) has an integral optimum solution, since its constraint matrix is totally unimodular. Let us now consider the dual linear program that reads

$$
\min \sum_{i=1}^{n}\left(u_{i}+v_{i}\right)
$$

subject to

$$
u_{i}+v_{j} \geq w_{i j}, \quad i, j=1, \ldots, n
$$


Let $u_{i}$ and $v_{i}, i=1, \ldots, n$, denote an optimum dual solution. Since matrix transposition preserves total unimodularity, we may assume that the solution is integral. Moreover, we may also assume that

$$
\min v_{j}=0
$$

since otherwise set $u_{i}^{\prime}:=u_{i}+\alpha, v_{i}^{\prime}:=v_{i}-\alpha$ where $\alpha:=\min v_{j}$. Let $j_{0}$ be the vertex with $v_{j_{0}}=0$. Then $u_{i}+v_{j_{0}} \geq 1-p$, and hence

$$
-u_{i}<p \quad \text { for every } i \text {. }
$$

Another immediate consequence of (7) is

$$
1-u_{i} \leq v_{j} \quad \text { for every } i j \in E .
$$

We will use the dual solution to define a vertex cut set $S$ in the network $N(G, p)$. Set

$$
S=\left\{i(t) \mid-u_{i}<t \leq v_{i}\right\} .
$$

Since $u_{i}+v_{i} \geq w_{i i} \geq 0$ there are at most $u_{i}+v_{i}$ vertices $i(t)$ for each $i$. Hence $|S| \leq \sum\left(u_{i}+v_{i}\right)=\max \sum w_{i j} x_{i j}$. We have to check that $S$ is a vertex cut set. For let $i_{0}, i_{1}, \ldots, i_{p}$ be a $p$-path in $N(G, p)$, and let $u_{0}, \ldots, u_{p}$ and $v_{0}, \ldots, v_{p}$ be the corresponding dual variables. Consider the minimum $t$ such that $t>-u_{t}$. Such a $t$ must exist by (9). If $t=0$, then $-u_{0}<0 \leq v_{0}$, and hence the vertex $i_{0}$ belongs to $S$. Assume $t>0$. By the choice of $t$, we have $-u_{t-1} \geq t-1$, or, equivalently, $t \leq 1-u_{t-1}$. Since the pair $i_{t-1} i_{t}$ forms an edge, we have by (10) that $-u_{t}<t \leq 1-u_{t-1} \leq v_{t}$. Hence $i_{t} \in S$ by (11). Since $S$ contains a vertex of each $p$-path, there are at most $|S|$ vertex disjoint $p$-paths in $N(G, p)$, and hence at most $|S|$ independent $p$-walks in $G$.

The opposite inequality is trivial. Given a cycle $C$ of $G$, we can define $|C|$ independent $p$-walks starting at distinct vertices of the cycle and walking in its direction. Given a path $P$ of length at least $p$, we can define $|P|-p$ independent $p$-walks starting at distinct vertices of the path but the last $p$ ones, and walking in the direction of the path. Thus a solution of the packing problem (2) with value $k$ yields $k$ independent $p$-walks.

We say that a matrix $A=\left(a_{i j}\right)$ of size $n$ by $n$ has the pattern $G$ if $a_{i j} \neq 0$ if and only if $i j$ is an edge of $G$.

Lemma 2. Let $G$ be a digraph and $p$ a positive integer. Then the maximum rank of the product of $p$ matrices $A_{1}, \ldots, A_{p}$ where each $A_{i}$ has the pattern $G$ equals the maximum number of independent p-walks in $G$.

It will be convenient to adopt some notation of [8]. Let $H$ be a digraph with weights $a_{e}, e \in E(H)$, on the edges. The weight of a subdigraph $H^{\prime}$ is defined as weight $\left(H^{\prime}\right):=\prod a_{e}$ where the product is over the edges of $H^{\prime}$. The weight of a family $\mathscr{H}$ of subdigraphs is defined as the sum of the weights of its members, i.e. weight $(\mathscr{H}):=\sum_{H^{\prime} \in \mathscr{H}}$ weight $\left(H^{\prime}\right)$. 
Proof. Let $A_{1}, \ldots, A_{p}$ be matrices of pattern $G$, and let $B=\left(b_{i j}\right)$ be their product. Let us consider $a_{i j, t}$, the nonzero $i j$ th entry of $A_{t}$, as the weight of the edge $(i j)_{t}:=i(t-1) j(t)$ of digraph $N(G, p)$. Then $b_{i j}=\operatorname{weight}\left(\mathscr{P}_{i j}\right)$ where $\mathscr{P}_{i j}$ is the collection of paths from $i(0)$ to $j(p)$ in $N(G, p)$. Let $k$ be the rank of $B$. Then $B$ has a nonsingular $k$ by $k$ submatrix $B_{R, C}$ with rows $R=\left\{r_{1}, \ldots, r_{k}\right\}$ and columns $C=\left\{c_{1}, \ldots, c_{k}\right\}$. We have

$$
\begin{aligned}
& \operatorname{det} B_{R, C}=\sum_{\pi} \operatorname{sg}(\pi) \text { weight }\left(\mathscr{P}_{r_{1} s_{\pi(1)}}\right) \cdots \operatorname{weight}\left(\mathscr{P}_{r_{k} s_{\pi(k)}}\right) \\
& =\sum_{\pi} \operatorname{sg}(\pi) \sum_{w_{r_{1} s_{\pi(1)}, \ldots,}, w_{r_{k} s_{\pi(k)}}} \prod_{i=1}^{k} \operatorname{weight}\left(w_{r_{i} s_{\pi(i)}}\right)
\end{aligned}
$$

where $w_{i j}$ is a path from $i(0)$ to $j(p)$ (or a $p$-walk from $i$ to $j$ in $G$ ). We say that the $p$-walks $w^{1}, \ldots, w^{k}$ form an $R-C$ system if the walks start and terminate in mutually distinct vertices of $R$ and $C$ respectively, i.e. $w^{1}=$ $w_{r_{1} s_{\pi(1)}}, \ldots, w^{k}=w_{r_{k} s_{\pi(k)}}$ for some permutation $\pi$. We say that an $R-C$ system is odd (even) if the permutation $\pi$ is odd (even), and we set $\operatorname{sg}\left(w^{1}, \ldots, w^{k}\right)$ $:=\operatorname{sg}(\pi)$. Let $H=H\left(w^{1}, \ldots, w^{k}\right)$ be the multi-digraph obtained by the union of edges of $w^{1}, \ldots, w^{k}$. ( $H$ may have multiple edges.) It is clear that the union of distinct $R-C$ systems may produce the same digraph $H$. Let even $(H)(\operatorname{odd}(H))$ be the number of even (odd) $R$ - $C$ systems whose union is $H$. Thus $H$ always has $k p$ edges. Now we have

$$
\operatorname{det} B_{R, C}=\sum(\operatorname{even}(H)-\operatorname{odd}(H)) \cdot \operatorname{weight}(H)
$$

where the sum is over all multi-subdigraphs of $N(G, p)$ with exactly $k p$ edges.

We observe that even $(H)=\operatorname{odd}(H)$ for every $H$ which is the union of an $R-C$ system $w^{1}, \ldots, w^{k}$ of $p$-walks that are not vertex independent. It is so because we have a vertex $v$ and edges $e, e^{\prime}$ from $v$ in $H$ such that any two walks $w$ and $w^{\prime}$ using $e$ and $e^{\prime}$ can be switched so that $w$ continues along $e^{\prime}$ and $w^{\prime}$ along $e$. This defines a 1-1 mapping between the odd and even $R-C$ systems whose union is $H$. On the other hand, the unions of distinct vertex independent $R-C$ systems are distinct. Thus we have

$$
\operatorname{det} B_{R, C}=\sum_{w^{1}, \ldots, w^{k}} \operatorname{sg}\left(w^{1}, \ldots, w^{k}\right) \cdot \operatorname{weight}\left(w^{1} \ldots w^{k}\right)
$$

where the sum is over all vertex independent systems of $k p$-walks which start in $R$ and terminate in $C$. Now Lemma 2 easily follows. If $\operatorname{det} B_{R, C} \neq 0$ there are $k$ vertex independent $p$-walks in $G$. Conversely, if there is such a system then let $R$ and $C$ denote the sets of origins and terminals of these $k$ walks, respectively. If the entries of $A_{1}, \ldots, A_{p}$ are chosen such that their union is algebraically independent, then $\operatorname{det} B_{R, C}$ is nonzero.

Let us note that if $A_{1}=A_{2}=\cdots=A_{p}=A$, it may still happen that

$$
\operatorname{weight}\left(w^{1} \bigcup w^{2} \bigcup \cdots \bigcup w^{k}\right)=\operatorname{weight}\left(\bar{w}^{1} \bigcup \bar{w}^{2} \bigcup \cdots \bigcup \bar{w}^{k}\right)
$$

for two distinct systems of vertex independent $p$-walks. 
Theorem 3. Let $G$ be a digraph and $p$ a positive integer. Then the maximum rank of the pth power of a matrix of pattern $G$ equals the maximum number of independent $p$-walks in $G$.

Proof. Clearly, $\max r A^{p} \leq \max r\left(A_{1} \ldots A_{p}\right)$ where all the matrices are of pattern $G$. Let $k$ be the maximum of the right hand side. Then there are $k$ independent $p$-walks in $G$ by Lemma 2 . By Theorem 1, there is a packing $\mathscr{F}$ of paths and cycles with value $k$ of the objective function (1). Let $w^{1}, \ldots, w^{k}$ be the independent $p$-walks defined by $\mathscr{F}$. Obviously, there is no other system of independent $p$-walks with the weight equal weight $\left(w^{1} \cup \cdots \cup w^{k}\right)$ even if $A_{1}=A_{2}=\cdots=A_{p}$. Thus $r A^{p}=k$ if algebraically independent transcendentals are substituted to nonzero entries of $A$.

The following statement has been formulated as a conjecture by $\mathrm{J}$. Holenda and M. Schlegel [4], who also proved the case $p=2$.

Corollary 4. The maximum possible rank of a product of $p$ matrices of the same pattern equals the maximum rank of the pth power of a matrix of the same pattern

If $p \geq n$, then the solution of the packing problem (2) consists of cycles only. Thus we have

Corollary 5. For any $p \geq n$, the maximum possible rank of the pth power of a matrix of the pattern $G$ equals the maximum number of vertices that can be covered by a family of vertex disjoint cycles. (Hence the solution is the same for all $p \geq n$.)

As a further application of Theorem 1 we show that Theorem 3 remains true even for symmetric matrices. If $A$ is a symmetric matrix, then more systems of $p$-walks may have the same weight, and in the consequence more terms in the determinant may cancel.

Corollary 6. Let $G$ be a symmetric digraph and $p$ a positive integer. Then the maximum possible rank of the pth power of a symmetric matrix of pattern $G$ equals the maximum number of vertices of $G$ that can be covered by a family of vertex disjoint cycles. (Hence the solution does not depend on $p$.)

Proof. Let us first observe the following fact. Let $C$ by a cycle in a symmetric digraph $G$, and let the vertices of $C$ be, say, $1,2, \ldots, s$. Denote by $\pi$ the permutation $(2,3, \ldots, s, 1)$. Assume that $s$ people are located in the vertices of the cycle, and at each time $t=1,2, \ldots, p$ all of them simultaneously walk either in the direction of $\pi$ or $\pi^{-1}$. Let $w^{1}, \ldots, w^{s}$, be the resulting $p$-walks. Then

$$
\text { weight }\left(w^{1} \bigcup \cdots \bigcup w^{s}\right)=(\text { weight } C)^{p}=\left(a_{12} a_{23} \ldots a_{s 1}\right)^{p} .
$$

Observe also that we describe the only way in which a system of independent $p$-walks of this weight may arise, provided $a_{i j}, j \geq i, i j \in E$, are algebraically 
independent. Since $\operatorname{sg} \pi=\operatorname{sg} \pi^{-1}$, we have $\operatorname{sg}\left(w^{1}, \ldots, w^{s}\right)=(\operatorname{sg} \pi)^{p}$. Thus the sign of any system of independent $p$-walks of weight equal to (weight $C)^{p}$ is the same, and hence their terms cannot cancel when computing the determinant of a submatrix.

Since $G$ is symmetric, we may clearly assume that the optimum solution of the packing problem consists of cycles only, say, $C_{1}, \ldots, C_{l}$. By the above argument, all systems of independent $p$-walks of weight that is equal to weight $\left(\left(C_{1} \cup \cdots \cup C_{1}\right)\right)^{p}$ have the same sign.

\section{CONCLUDING REMARKS}

The main result of the paper is Theorem 3 which establishes the maximum possible rank of powers of a matrix of a given pattern. Let us recall that the case $p=1$ has been considered in [2]. Also the case $p=2$ seems to be simpler than the general case. The solution has been presented in [4], and $J$. Nešetřil communicated to me that it was known also to $\mathrm{Ch}$. Papapdimitriou. Theorem 3 for $p=2$ follows also from the fact, that if two systems of independent 2-walks have the same weight, then they have also the same sign. This fact is not true for $p \geq 3$.

The proof of Theorem 1 based on the duality of linear programming and the total unimodularity is in the spirit of [3]. Let us note that one can also derive a direct combinatorial proof using some augmenting configurations. However, the proof presented here provides also some information about possible vertex cut sets in the associated network $N(G, p)$.

Let $A(x)$ be the matrix whose $i j$ th entry is the variable $x_{i j}$ for $i j \in E$ and it is zero otherwise. Let $p$ be a positive integer, $B(x)$ denote the $p$ th power $A^{p}(x)$ of $A(x)$, and let $D(x)$ be the determinant of a $k$ by $k$ submatrix of $B(x)$. Clearly, $D(x)$ is a polynomial in the variables $x_{i j}$. I propose the following question. What is the complexity of computing a coefficient of a given monomial of $D(x)$ ?

Consider the following modified question. Given a digraph $G$, let $A$ be a matrix whose $i j$ th entry may be nonzero only if $i j$ is an edge but the converse is not required. Clearly, the maximum possible rank of a power of such a matrix is also given by Theorem 3 . We show that the maximum is achieved already by a 0,1 -matrix. Let $\mathscr{F}$ be an optimum solution of the packing problem (2). Then the maximum rank is realized by setting $a_{i j}=1$ if $i j$ is an edge of a member of $\mathscr{F}$ and $a_{i j}=0$ otherwise. We mention that the statement remains true for a matrix over an arbitrary field. (I am indebted for the question concerning other fields to A. Faragó.)

The results presented in the paper have been announced in [6]. Further combinatorial aspects of Theorem 1 and some application to automatic control will appear elsewhere. 


\section{ACKNOWLEDGMENTS}

I thank F. Barahona who noticed that it is more straightforward to formulate (4) as an assignment problem, instead of a flow circulation used earlier. I also thank J. Kratochvil for a valuable discussion.

\section{REFERENCES}

1. C. Berge, Graphs and hypergraphs, North-Holland, 1973.

2. J. Edmonds, Systems of distinct representatives and linear algebra, J. Res. Nat. B.S. B71 (1967), 233-240.

3. A. J. Hoffman, The role of unimodularity in applying linear inequalities to combinatorial the orems, Annals of Discrete Math. 4 (1979), 73-84.

4. J. Holenda and M. Schlegel, lecture presented at Czechoslovak Conference on Combinatorics, 1987.

5. J. E. Hopcroft and R. M. Karp, A $n^{5 / 2}$ algorithm for maximum matchings in bipartite graphs, SIAM J. Comp. 2 (1973), 225-231.

6. S. Poljak, An announcement, Comm. Math. Univ. Car. 29, 1 (1988).

7. A. Schrijver, Theory of linear and integer programming, Wiley, 1986.

8. D. Zeilberger, A combinatorial approach to matrix algebra, Discrete Math. 50 (1985), 61-72.

Charles University, Faculty of Mathematics and Physics, Department of Applied Mathematics, Malostranské n.25, 11800 Praha 1, Czechoslavakia 